\title{
Functional Parcellation of Memory Related Brain Networks by Spectral Clustering of EEG Data
}

\author{
Cagatay Aydin, Oytun Oktay, Adem Umut Gunebakan, Rifat Koray Ciftci, and Ahmet Ademoglu
}

\begin{abstract}
In this study, we investigate the clustering information of alpha band brain networks during memory load task. For this purpose, short time memory task which includes memory load varieties is implemented to the subjects. To calculate mutual information, time and frequency information is both taken into consideration due to Cohen class time-frequency distribution (TFD) formulation. Cohen class based mutual information helps us to integrate adjacency matrices based on the similarity information of individual electrode pairs. In addition, essential frequency bins are selected from the TFD with respect to the default alpha frequency $(8-12 H z)$ intervals. Moreover, graph based spectral clustering algorithm is used to parcellate memory related circuits on the brain. From the calculated adjacency matrices, the N-cut algorithm is used for node wise clustering between nodes. After node wise clustering information, subject wise clustering is applied with respect to the similarities of node information over all subjects.
\end{abstract}

Keywords-EEG, Memory Load, Mutual Information, Normalized Cut, Working Memory

\section{INTRODUCTION}

$\mathbf{H}$ ANS Berger was the first person to perform electroencephalographic scalp recordings and found an intermediary device, the human Electroencephalogram (EEG). The use of EEG moved into multidisciplinary areas such as Psychiatry and Neurology. The brain abnormalities such as epilepsy and structural lesions were some of the major fields in clinical applications [1]. The EEG signal and its oscillatory activity are strongly related with temporal modulation of information processing [21]. Generally, oscillations of alpha $(8-12 \mathrm{~Hz})$ band of the EEG rhythm and the amplitude of the signal changes in the memory and cognitive based tasks are observed [7], [6]. Thus, determining shorter time scale analysis of the EEG signal, information theoretic measures may provide enhanced results to observe memory related brain regions.

Manuscript received April 14, 2012. This study is supported by Scientific and Technological Research Council of Turkey (TÜBİTAK) under project number 109E202.

C. Aydin is with the Institute of Biomedical Engineering, Bogazici University, Kandilli Campus, Cengelkoy/ Istanbul, 34684 TURKEY Phone: +90 533-4371156 e-mail: cagatay.aydin@boun.edu.tr

O. Oktay is with the Electronics and Communications Engineering Department, Namik Kemal University, Corlu/ Tekirdag, 59860 TURKEY e-mail moktay@nku.edu.tr

U. Gunebakan is with the Institute of Biomedical Engineering, Bogazici University, Kandilli Campus, Cengelkoy/ Istanbul , 34684 TURKEY e-mail: adem.gunebakan@boun.edu.tr

K. Ciftci is with the Biomedical Engineering Department, Namik Kemal University, Corlu/ Tekirdag, 59860 TURKEY e-mail: kciftci@nku.edu.tr

A. Ademoglu is with the Institute of Biomedical Engineering, Bogazici University, Kandilli Campus, Cengelkoy/ Istanbul, 34684 TURKEY e-mail: ademoglu@boun.edu.tr
There is much evidence that, the brain deals with real time information from the rapid interactions between sensory channels and well specified brain regions [2]. The sophisticated architecture of brain networks can be observed with the help of spectral clustering algorithm to create the dynamic interactions between regions like clusters and the directions of information flow from one cluster to another.

Several strategies have been proposed for investigating the memory processing in the brain. Basar et al. suggested that the alpha band and its oscillatory components could be a bridge between the thalamus and the cortex, which was affected by the synchronized neural activity. Furthermore, Klimesh et al. came up with investigating memory related brain dynamics with respect to dynamic property of memory processing. Following their assumption that the memory processing is using the longitudinal pathways (feedback loops) linking the thalamic nuclei with the cortex [3], one of the most predominant rhythms (alpha $8-12 \mathrm{~Hz}$ ) can be used to extract the memory information in these pathways. Additionally, by implementing frequency analysis, they found a significant difference in the alpha rhythm between subjects who had a bad and good memory performance. The relation between the alpha band and the memory related brain dynamics was investigated by using the amplitude analysis of the alpha signal in terms of the overall power distribution of the signal and the shifts in the power. Furthermore, these shifts came with an event-related desynchronization (ERD) term which was first found by Pfurtscheller et al. The main idea of ERD relies on a percentage difference which can be determined as an increase or decrease in the band power during a test interval with respect to the reference interval. During retrieval period of the memory task, bad memory performers showed a lack of significant desynchronization in the lower alpha $(8-10 \mathrm{~Hz})$.

Some particular studies aimed to understand the structure of the brain circuitry during memory load. Jensen et al. used modified STM paradigm (Stenberg task) for this purpose. Moreover, their experiment design allowed them to separate the encoding, the retention and the recognition states of the memory processing. They found increased peak in the alpha oscillations due to an increased memory load. They assumed that power increase in the alpha oscillations reflected the synchronization across multiple brain regions to achieve active inhibition [4]. To find out working memory related brain circuits Sauseng et al. [6] used an experimental design which investigated the differences between retention and manipulation organizations in the brain. They found a strong prefrontal alpha power increase and an occipital alpha suppression during visuospatial information processing. Fur- 
A
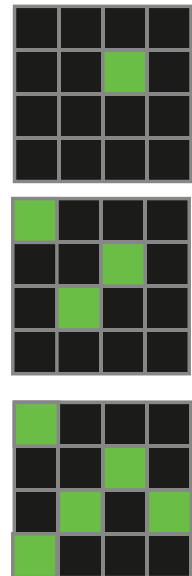

Memory Set Retention $500 \mathrm{~ms}$ $2500 \mathrm{~ms}$
$1 \mathrm{BOX}$

$3 \mathrm{BOX}$
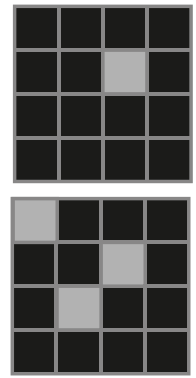

5 BOX

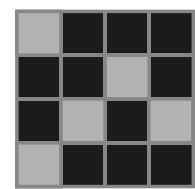

Probe \& Response

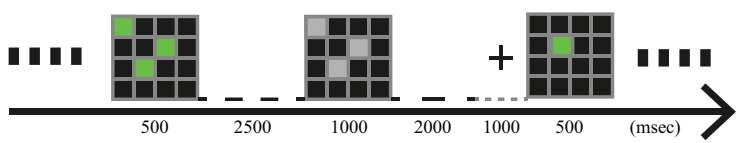

C
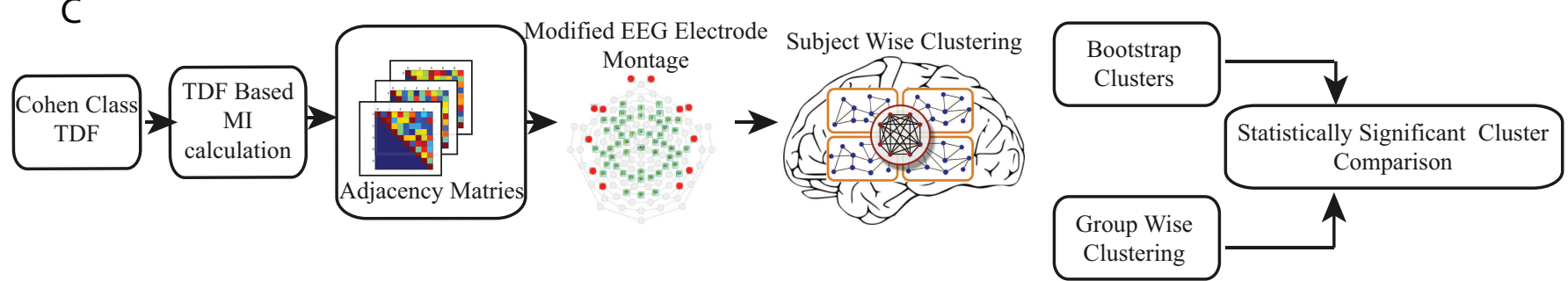

Fig. 1. A; The representation of the visuospatial memory task, B; Topological view of EEG electrode montage locations and red dots are used to initialize the electrode elimination process, C; Flow diagram of the entire analytical process

thermore, in the memory scanning task, an alpha band specific event related synchronization (ERS) was observed during the retention interval of the memory load based experiment [7]. In other words, retention interval can be referred to as the time spent after certain encoded items were seen by the subject and kept by the encoded information in mind. In the experiment, they observed an increase in the alpha band power by the increased number of represented items.

Estimating the time delays between EEG electrodes, Shannon entropy based mutual information calculation (MI) was used by Moddemeijer et al [8]. In order to investigate both the time and the frequency components of an EEG signal by mutual information analysis, time-frequency distributions (TFD) are consistent measures. MI generally represents a measure of independence between random variables. Hence, to generate the statistical difference between signal pairs, individual TFDs and a joint TFD of a signal pair is adequate enough to calculate the time-frequency based MI [11], [12]. Lu et al. used the time-frequency cross MI analysis to inquire the functional connectivity in the alpha and in the beta bands [13]. Spectral clustering has manifest advantages among varieties of clustering methods due to its implementation ease, calculation efficiency and enhancement of traditional clustering algorithms such as k-means. Furthermore, the second eigenvector of the Laplacian matrix had an important role on bi-partitions of the graph [14]. Shi et al. referred to a normalized cut algorithm as an unbiased measure which allowed to establish a sub-group of a graph by minimizing normalized cut with maximizing the similarity within the cluster [15]. There are several fMRI studies which have observed the resting state network properties [16], [18], and brain tumor separation [17], by using the n-cut algorithm.

\section{Methods}

\section{A. Experiment Design}

1) Participants: In this experiment, the EEG data was recorded over 17 (13 male, 5 female mean age of 23) healthy subjects. Experimental procedure was applied as voluntarily and participants signed an informed consent about their medical history in order to exclude those subjects who might have brain related disorders.

2) Task: The visuospatial short time memory task (Figure 1 A) was run in the ongoing experiment. The locations of one, three or five targets on $4 \times 4$ box model were shown to the subjects for 400 milliseconds. On each stimulus, one of three box combinations (one, three or five box) was represented randomly. Due to the box combination model, their positions had to be kept in memory for 2500 milliseconds and had to be compared via answer stimulus which was presented in gray color. A subject should respond to the answer stimulus whether 
the target box positions were stated as exactly the same as answer box positions. After 2,500 milliseconds of retention interval, if it was true, subjects had to answer with the right mouse button press or vice versa in 1000 milliseconds. To be able to preserve the event synchronization, the recording and the experiment computers were synchronized after each trial by using their clocks.

The segmentation period for this experiment was $4000 \mathrm{~ms}$. Each segment started from 500ms after the probe (green) stimulus to $1000 \mathrm{~ms}$ after the answer stimulus (gray). The significant time interval for each segment which was 2500 $\mathrm{ms}$ long between the probe and the answer, was used in analysis. Furthermore, the main reason for initializing longer time interval in segmentation was to secure the signal from fluttering in the filtering of preprocessing stages. Each answer, each reaction time, and the overall mean reaction time for each box model were calculated.

There were 100 stimuli for each box combinations and there were equal number of true and false probes. In total, 300 stimuli were represented during the experiment. Only correct answers were taken into consideration. The experimental design was made by the Psyctoolbox, Matlab software.

3) Data Acquisition and EEG Preprocessing: EEG recordings were done by 64 channel EGI HydroCel amplifier.

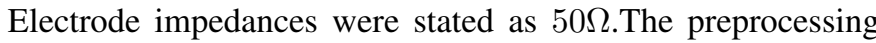
configuration can be listed as; $0.1 \mathrm{~Hz}$ first order high pass filter, $100 \mathrm{~Hz}$ low pass filter, $50 \mathrm{~Hz}$ Notch filter, segmentation of correct segments, artifact detection tool, ocular artifact removal tool, bad channel replacement tool and file export tool. All of preprocessing routine was done with EGI Net Station software tools.

\section{B. Information Theoretical Analysis on Time-Frequency Plane}

To calculate both time and the frequency components of the EEG segments, Cohen class distributions were calculated. The main formula of Cohen's class $C(t, f)$ can be expressed as;

$$
\begin{aligned}
C(t, f)= & \left(\iiint \phi(\theta, \tau)\right. \\
& \left.s\left(u+\frac{\tau}{2}\right) s^{*}\left(u-\frac{\tau}{2}\right) e^{j(\theta u-\theta t-2 \pi \tau f)} d u d \theta d \tau\right)
\end{aligned}
$$

The $s$ represents the signal and the $\phi(\theta, \tau)$ stands for the kernel function. Energy preservation is an important property of TDFs which can be satisfied when $\phi(\theta, 0)=\phi(\theta, \tau)=$ $1 \forall \tau, \theta$. Then, the equation can be represented as;

$$
\begin{gathered}
\iint C(t, f) d t d f=\int\left|s(t)^{2}\right| d t=\int\left|S(f)^{2}\right| d f \\
\int C(t, f) d f=\left|s(t)^{2}\right|, \int C(t, f) d t=\left|S(f)^{2}\right|
\end{gathered}
$$

The adaptation of entropy and other information theoretical measures can be expressed with the help of Equation 2 and Equation 3. Probability density functions (PDFs) and timefrequency distributions (TFDs) are different from each other that, TFDs are not always positive like PDFs. For each memory load condition, subject-wise TFDs are calculated with the help of equation 3 by combining all related frequency bins which are precise alpha bins between 8 and $12 \mathrm{~Hz}$. Cohen class TFDs are calculated by using TFTB, Matlab software.

\section{Mutual Information Calculation}

For a brief approximation of one dimensional mutual information (MI) calculation, assume that two random variables $X$ and $Y$ have mutual information which can be expressed as;

$$
I(X ; Y)=\sum_{x} \sum_{y} p(x, y) \log \frac{p(x, y)}{p(x) p(y)}
$$

The $p(x, y)$ is joint probability density function and, $p(x)$ and $p(y)$ are marginal PDFs of $X$ and $Y$. If $X$ and $Y$ are independent from each other, $\mathrm{MI}$ is determined as minimum and equal zero.

To calculate the Cohen class TFD with the help of Cohen class distribution, energy density functions are taken into account instead of PDFs. If we replace the marginal densities $p(x), p(y)$ with the individual energy densities $C_{x}(t, f), C_{y}(t, f)$ and the joint PDFs $p(x, y)$ with the joint energy distributions $C_{x y}(t, f)$, the MI equation can be referred to as;

$$
I\left(C_{x}, C_{y}\right)=\iint\left|C_{x y}(t, f)\right| \log \frac{\left|C_{x y}(t, f)\right|}{C_{x}(t, f) C_{y}(t, f)} d t d f
$$

Mutual information is calculated by the Equation 5 to generate adjacency matrices.

\section{Subject-wise Clustering}

$\mathrm{N}$-cut clustering is an algorithm which computes an unbiased measure that allows to establish a sub-group of a graph by minimizing the normalized cut and maximizing the similarity within the cluster. In contrast to other graph clustering methods, it does not make strong assumptions on outliers which can be considered as an important advantage. From the EGI EEG amplifier default electrode locations, the red dotted electrodes are eliminated [Figure $1 \mathrm{~B}$ ]. In other words, to increase the clustering significance, eye and ear electrodes are removed. N-cut clustering algorithm ([15] see Appendix A) is applied on each subject. The number of clusters is set to 4 in subject-wise analysis.

\section{E. Group-wise Clustering}

To implement group-wise analysis, an adjacency matrix of overall subjects is calculated due to the similar clustering information of each subject's clusters. Furthermore, the procedure of $\mathrm{N}$-cut clustering algorithm is repeated over the generated adjacency matrix. The clustering information is topologically plotted over modified electrode locations to investigate the comparison between different box models [Figure 2; A,B,C]. As same as subject-wise clustering analysis, four cluster based parcellation is taken into consideration.

In order to achieve statistically significant electrodes from group-wise clusters, bootstrap technique is employed [19]. A 

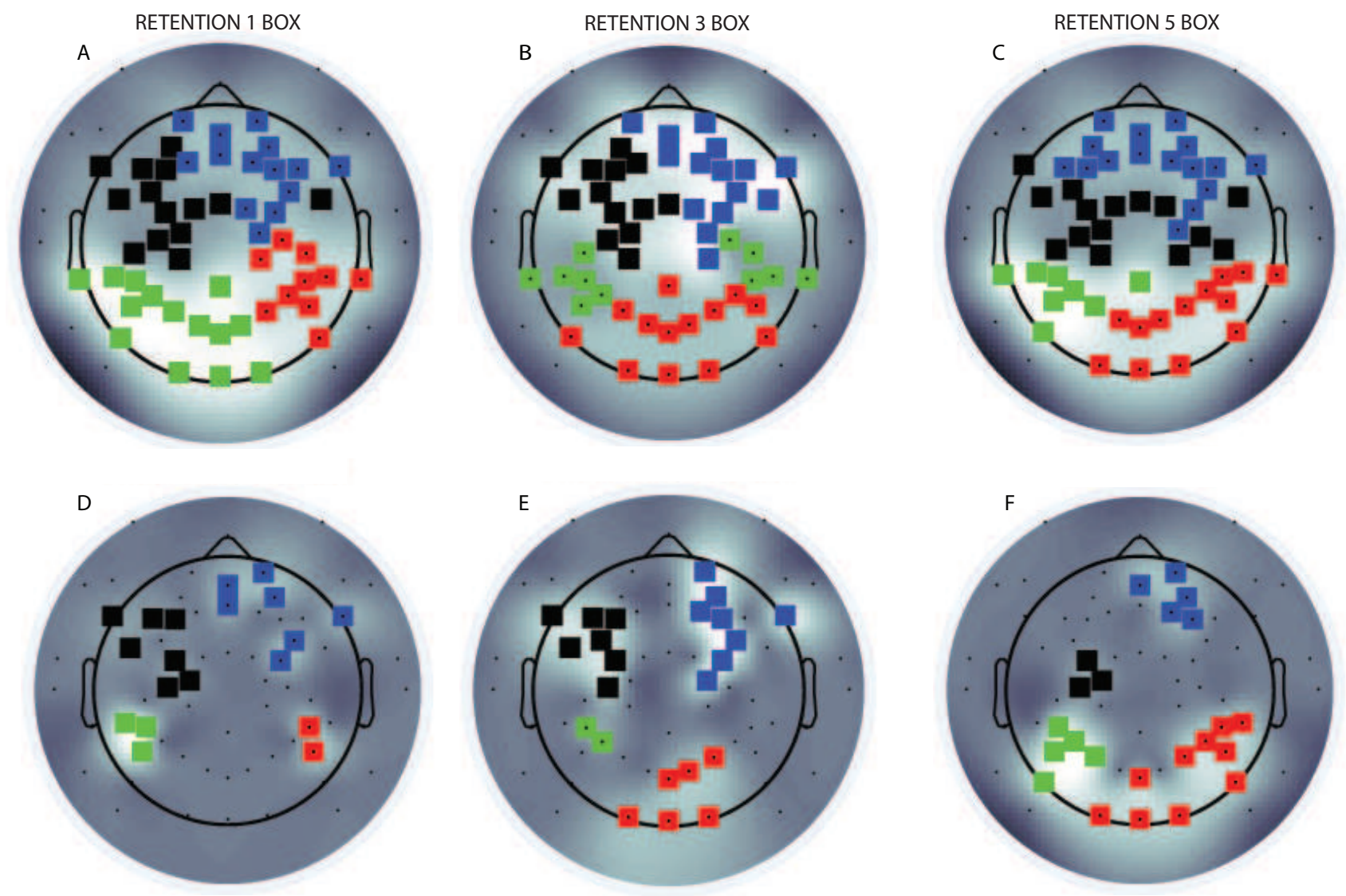

Fig. 2. Topological representation of alpha band $(8-12 \mathrm{~Hz})$ clusters, A,B, $\mathrm{C}$ are representing the clusters during the retention period of 1 box, 3 box and 5 box models, D,E,F are representing the statistically significant nodes within clusters during above column-wise retention models.

simulation database is generated by mimicking the subjectwise clusters to test the significance of group-wise clusters. Over 17 subject-wise clusters, 1000 pseudo subject-wise cluster combinations are generated. Pseudo combinations which can comprise repetitive sequences are randomly distributed. After the generation of simulation database, $\mathrm{N}$-cut algorithm is employed over each combination to achieve 1000 group-wise clusters. Overall mean is determined from calculated groupwise clusters and significant nodes within clusters which are larger than $\% 95$ are selected [Figure 2; D,E,F]. To obtain topological representation EEGLAB, Matlab software is used.

\section{RESULTS}

\section{A. Behavioral Results}

The percentage of correct responses and the mean reaction time of all subjects were computed to compare the difference between 3 task conditions. There was no statistically significant difference in correct responses between 1 box $(\% 94,8 \pm 4,0)$ and 3 box model $(\% 93,13 \pm 5,3)(p=0,054)$ and between 3 box and 5 box model $(\% 92,9 \pm 4,6)(p=$ $0,811)$. However, there was a significant difference between 1 box and 5 box model $(p=0,0030)$.

Directly proportional reaction time increase was observed by the number of represented boxes. The mean reaction time due to box models can be listed as follows; 1 box model $0,50 \pm$ $0,07,3$ box model $0,53 \pm 0,08,5$ box model $0,55 \pm 0,08$ seconds.

\section{B. Experimental Results}

In Figure 2, modified electrode locations obtained by removing face and ear electrodes from default electrode locations are shown. Their clustering information, and the statistically significant electrode node locations within their clustering information are plotted over topographic maps. During 1 box retention period [Figure 2; A,D], several prefrontal and left parietal electrodes are observed within two separate cluster groups due to a statistically significant map. In addition, there are slightly small occipital clusters which are generally grouped around the left and the right occipital region of the brain. During 3 box retention period [Figure 2; B,E], statistically significant electrodes were located over the left and the right parietal region and the number of the electrodes in occipital region were increased. However, during 5 box retention period [Figure $2 ; \mathrm{C}, \mathrm{F}$ ], statistically significant electrodes formed a larger cluster which could be observed as a combination of the left parietal and the occipital region of the brain due to the increased memory load. Furthermore, various 
significant nodes and small group of clusters were located in left parietal and prefrontal regions.

\section{Discussion AND CONCLUSIONS}

In this study, we investigated the clustering information of memory related brain networks. There are several alpha band related studies with respect to the memory load and its reflection on the alpha band. Tuladhar et al. investigated that, a parametric increase in the alpha band activity over posterior brain areas were observed with the memory load [20]. Jensen and his colleagues assumed that there could be separate memory related sources which were located on the posterior and bilateral regions of the brain [4]. In addition, they inferred that the memory load activity in the separate brain regions could reflect the degree of synchronization across multiple brain regions involved. As shown in Figure 2, modified electrode locations and their statistically significant nodes provide support for the previous studies. In other words, when the number of boxes increases, the size of clustered nodes which are projected on the occipital and bilateral regions increases. Furthermore, the left parietal and occipital clusters in [Figure 2; D,E,F], merged into a larger cluster which may indicate the increased memory load.

\section{APPENDIX A \\ N-CUt Clustering Algorithm}

The size of the partition can be described as; $|A|=$ the number of vertices in $A$

$$
\operatorname{vol}(A)=\sum_{i \in A} d i
$$

Number of vertices is used to measure the size of partition A. On the other hand, $\operatorname{vol}(A)$ measures the size in terms of summation over the weights of all edges which are attached to vertices in $\mathrm{A}$.

$\mathrm{N}$-cut algorithm can be described as;

$$
\begin{gathered}
\operatorname{cut}(A, \bar{A})=\sum_{v_{i}=A, v_{j}=\bar{A}} w(i, j) \\
\operatorname{Ncut}(A, \bar{A})=\frac{\operatorname{cut}(A, \bar{A})}{\operatorname{Vol}(A)}+\frac{\operatorname{cut}(A, \bar{A})}{\operatorname{Vol}(\bar{A})}
\end{gathered}
$$

According to Shi and Malik, normalized spectral clustering algorithm can be listed as follows;

- The similarity matrix has to be generated and the number of $k$ clusters has to be indicated,

- With the help of weighted graph, $W$ adjacency matrix is calculated,

- The unnormalized Laplacian has to be computed $L=$ $D-W$,

- Due to the number of $k$ clusters, eigenvectors have to be computed $u_{1}, \ldots u_{k}$ with the help of generalized formula $L u=\lambda D u$,

- The vector which consists of cluster points $\left(Y_{i}\right)_{i}=$ $1, \ldots, n$ is clustered with the help of $k$-means algorithm [14].

\section{REFERENCES}

[1] Boutros, N., and S. Galderisi, "Standard electroencephalography in clinical psychiatry: a practical handbook," Acta Psychiatrica Scandinavica, Vol. 124, no. 3, pp. 239-240, 2011.

[2] Sporns, O., and C. J. Honey, "Small worlds inside big brains.," Proceedings of the National Academy of Sciences of the United States of America, Vol. 103, pp. 19219-20, Dec. 2006.

[3] Klimesch, W., "EEG-alpha rhythms and memory processes.," International journal of psychophysiology : official journal of the International Organization of Psychophysiology, Vol. 26, pp. 319-40, June 1997.

[4] Jensen, O., J. Gelfand, J. Kounios, and J. E. Lisman, "Oscillations in the alpha band (9-12 Hz) increase with memory load during retention in a short-term memory task.," Cerebral cortex (New York, N.Y. : 1991), Vol. 12, pp. 877-82, Aug. 2002.

[5] Fink, a., R. H. Grabner, C. Neuper, and a. C. Neubauer, "EEG alpha band dissociation with increasing task demands.," Brain research. Cognitive brain research, Vol. 24, pp. 252-9, July 2005.

[6] Sauseng, P., W. Klimesch, M. Doppelmayr, T. Pecherstorfer, R. Freunberger, and S. Hanslmayr, "EEG alpha synchronization and functional coupling during top-down processing in a working memory task.," Human brain mapping, Vol. 26, pp. 148-55, Oct. 2005.

[7] Klimesch, W., "EEG alpha and theta oscillations reflect cognitive and memory performance: a review and analysis.," Brain research. Brain research reviews, Vol. 29, pp. 169-95, Apr. 1999.

[8] Moddemeijer, R., "On estimation of entropy and mutual information of continuous distributions," Signal Processing, Vol. 16, no. 3, pp. 233248, 1989.

[9] Rosso, O., M. Martin, and a. Plastino, "Brain electrical activity analysis using wavelet-based informational tools," Physica A: Statistical Mechanics and its Applications, Vol. 313, pp. 587-608, Oct. 2002.

[10] Schlogl, a., C. Keinrath, R. Scherer, and P. Furtscheller, "Information transfer of an EEG-based brain computer interface," First International IEEE EMBS Conference on Neural Engineering, 2003. Conference Proceedings., no. 1 0, pp. 641-644, 2003.

[11] Aviyente, S., "Information-theoretic signal processing on the timefrequency plane and applications," in Proc. of EUSIPCO, pp. 4-8, Citeseer, 2005.

[12] Aviyente, S., "A measure of mutual information on the time-frequency plane," in Acoustics, Speech, and Signal Processing, 2005. Proceedings.(ICASSP'05). IEEE International Conference on, Vol. 4, pp. iv-481, IEEE, 2005.

[13] Lu, C.-F., S. Teng, C.-I. Hung, P.-J. Tseng, L.-T. Lin, P.-L. Lee, and Y.$\mathrm{T}$. Wu, "Reorganization of functional connectivity during the motor task using EEG time-frequency cross mutual information analysis.," Clinical neurophysiology : official journal of the International Federation of Clinical Neurophysiology, Vol. 122, pp. 1569-79, Aug. 2011.

[14] Luxburg, U., "A tutorial on spectral clustering," Statistics and Computing, Vol. 17, pp. 395-416, Aug. 2007.

[15] Shi, J., and J. Malik, "Normalized cuts and image segmentation," Pattern Analysis and Machine Intelligence, IEEE Transactions on, Vol. 22, no. 8, pp. 888-905, 2000.

[16] Shen, X., X. Papademetris, and R. T. Constable, "Graph-theory based parcellation of functional subunits in the brain from resting-state fMRI data.," NeuroImage, Vol. 50, pp. 1027-35, Apr. 2010.

[17] Chen, V., and S. Ruan, "Graph Cut Based Segmentation of Brain Tumor From MRI Images," Vol. 3, no. December, pp. 1054-1063, 2009.

[18] van den Heuvel, M., R. Mandl, and H. Hulshoff Pol, "Normalized cut group clustering of resting-state FMRI data.," PloS one, Vol. 3, p. e2001, Jan. 2008.

[19] Bellec, P., V. Perlbarg, and A. C. Evans, "Bootstrap generation and evaluation of an fMRI simulation database.," Magnetic resonance imaging, Vol. 27, pp. 1382-96, Dec. 2009.

[20] Tuladhar, A. M., N. ter Huurne, J.-M. Schoffelen, E. Maris, R. Oostenveld, and O. Jensen, "Parieto-occipital sources account for the increase in alpha activity with working memory load.," Human brain mapping, Vol. 28, pp. 785-92, Aug. 2007.

[21] Busch, N. a., J. Dubois, and R. VanRullen, "The phase of ongoing EEG oscillations predicts visual perception.," The Journal of neuroscience the official journal of the Society for Neuroscience, Vol. 29, pp. 7869-76, June 2009. 